\title{
Towards Automated Business Process Deduction through a Social and Collaborative Platform
}

\author{
Aurélie Montarnal, Anne-Marie Barthe-Delanoë, Frédérick Bénaben, \\ Matthieu Lauras, and Jacques Lamothe \\ Mines Albi- University of Toulouse, Campus Jarlard, \\ Route de Teillet, 81000 Albi, France \\ \{aurelie.montarnal, anne-marie.barthe, frederick.benaben, \\ matthieu. lauras, jacques. lamothe\} @mines-albi.fr
}

\begin{abstract}
The French project OpenPaaS aims at providing a social and collaborative platform that supports inter-organizational collaborations. This platform is a social network in which subscribing organizations are defined by their profiles (i.e. their capabilities). In response to a collaboration opportunity suggested by an organization, the IT system intends to automatically deduce a corresponding inter-organizational process, meaning that it enables simultaneously (i) the discovery of the collaborative partners and (ii) the building of a collective business process model. This paper aims at describing the interactions that take place first, between the system and the users and second, within the system between the IT components. It also provides an overview of two specific reconciliations in the context of such a design of emerging networks: (i) functional versus non-functional and (ii) business versus technical.
\end{abstract}

Keywords: Inter-organizational collaboration, interoperability, business process deduction, ontology.

\section{Introduction}

Inter-organizational collaborations have dramatically increased these last years in various fields. The Virtual Organization (VO) concept has emerged as an IT solution for supporting inter-organizational collaborations. Two main dimensions gave rise to the virtual networks of organizations. On the one hand, Camarinha-Matos et al. [1] explain the concept of virtual networks of organizations by faster demands from customers and more complexity concerning products and services. On the other hand, Byrne [2] considers the VO as a crucial factor for a company to increase its competitiveness by quickly adapting to new business contexts and also promptly responding to new tenders. He describes the virtual corporation as a temporary alliance dedicated to fulfill a market opportunity. In this context the ability of the IT system to support efficient interactions between the organizations has become the main key factor for success. 
The OpenPaaS ${ }^{1}$ project aims at providing a platform to support fast business interactions between subscribing organizations. This platform provides a design time on which the inter-organizational collaborative process is deduced and transformed into a technical and orchestrable process (i.e. the automation of the two first steps of the lifecycle) and a run time that provides a support to the third step by orchestrating the process. The design time often turns out to be a manual and time-consuming task. Moreover it deals with competitive contexts in which the broker wants to find the best partners corresponding to its non-functional expectations (e.g. price, delivery time, geographical location...). This paper focuses on the design time that can be divided as follows: (i) knowledge gathering regarding the objective of the collaboration, (ii) exploration of the potential partners and, simultaneously, (iii) selection of the "best" partners and (iv) building of the corresponding "best" inter-organizational process.

This paper mainly aims at giving a state of the research concerning the knowledge gathering and the automated building of a process. From these two steps, it gives research directions to simultaneously reconcile process building and the selection of the partners. Section 2 focuses on a state of the art about the support of business process through a collaborative platform, then business process deduction and partners selection. Then Section 3 provides a functional solution for the business process deduction. Finally Section 4 aims at providing a discussion and further research works to extend the latter solution on a two axis reconciliation: functional/non-functional and business/technical.

\section{State of the Art}

\subsection{Business Process Support on Collaborative Platforms}

Facilitating business process modeling is a major priority that has been widely exploited these last years. A lot of products have been implemented in order to answer efficiency issues within a company (e.g. Bonitasoft). Closer to the inter-organizational collaborations problematic, some products like Kahua [3], ARIS [4] or SAP Netweaver [5] propose industrial platforms that enable organizations to share processes, workflows and also to exchange data.

With the XaaS (Everything as a Service) technologies emergence, BPaaS (Business Process as a Service) softwares have been created. According to Accorsi [6] $\mathrm{BPaaS}$ enables several organizations to work together on a process from design time to run time. Han et al. [7] consider the BPaaS as a scalable solution in that sense that companies pay regarding their use of the software. Moreover it increases the interoperability and the collaborations of the user companies by enabling them to share their own processes.

However these solutions come more as a way for supporting the coordination of the inter-organizational collaborations: the user companies have to design the concerned collaborative process before any orchestration and the partners know they are

\footnotetext{
1 https: / /research. linagora.com/display/

openpaas / Open+PAAS+Overview
} 
going to work together on a specified collaborative project. Thus the business process modeling takes a lot of time and relies on accurate knowledge about the collaboration (i.e. partners, sequence of activities...). In order to improve the flexibility of collaborative platform and to offer the users more efficiency, the deduction of the inter-organizational business processes becomes a necessity.

\subsection{Business Process Deduction}

Various frameworks have been established towards process deduction. EnterpriseReference Architecture and Methodology: GERAM [8] proposes a framework to support enterprise engineering and maintaining. It is specifically process oriented when detailing the lifecycle of enterprises. The difficulty for reusing this work in OpenPaaS relies on the fact that the concepts are too abstract. In [9], Mu proposes a framework based on two types of knowledge: (i) about the partner and (ii) about the collaboration to be set up. The goal is to help organizations that wish to work together to automatically design a business process that responds to the collaborative objectives. First, the partners describe their capabilities; second they propose a new collaboration objective. Thanks to the framework that has been established, from these two kinds of models, the IT system is able to deduce a process cartography that uses the capabilities of the partners. The further research works are mainly based on this framework, however it does not fully respond to the OpenPaaS needs since the partners of the collaboration are not already known. This is why an additional layer should be added to this framework to provide the discovery of the potential partners and then their final selection.

\subsection{Partners Selection}

In [10], Kulvatunyou et al. describe the use of a semantic-based framework which aims at deducing a distributed process plan that fulfills a specific collaborative goal. Nevertheless, this framework relies on a resource independent model initially given by the broker of the collaboration. The system focuses essentially on the discovery and filtering of the partners. Each partner is described through its profile that lists its capabilities. The selection of the final partners of the collaboration is based on the simulation and the evaluation of each potential manufacturers dependent process plan. Sha and Che [11] describe a genetic algorithm to select the partners of a complex supply chain. The algorithm seems very efficient but is also based on the fact that the main steps of the supply chain are already known.

\section{Process Deduction}

The deduction of an inter-organizational process is entirely based on semantic links provided by two ontologies: a Collaborative Ontology (CO) that provides generic objectives of collaboration and the capabilities to set up in order to reach the corresponding objective; and a Business Field Ontology (BFO) that specifies the domain of 
the collaboration. These ontologies allow the system to understand the information given by the users of the platform.

1. Modeler level (cf. Fig. 2 left side): first, organizations have to describe themselves in their profiles. These profiles contain all the capabilities (i.e. business services) that an organization can provide. These capabilities are linked with near by/same as relationships to the CO. Inputs and outputs should also be described and linked with hasBusinessDomain relationships to the BFO. Then, an objective of collaboration can be proposed in the same way, the broker should link it to the two ontologies.

2. Process deduction level (cf. Fig. 2 right side): two filters finally provide all the capabilities of the subscribing organizations to invoke in the final collaborative process: FI (cf. Fig. 2) allows keeping the capabilities that meet the objective of collaboration (via CO), and then FII determines the capabilities that also respect the right business domain. Thus, this latter set of capabilities can be ordered into a collaborative process by working on the inputs and outputs equivalence between the capabilities.

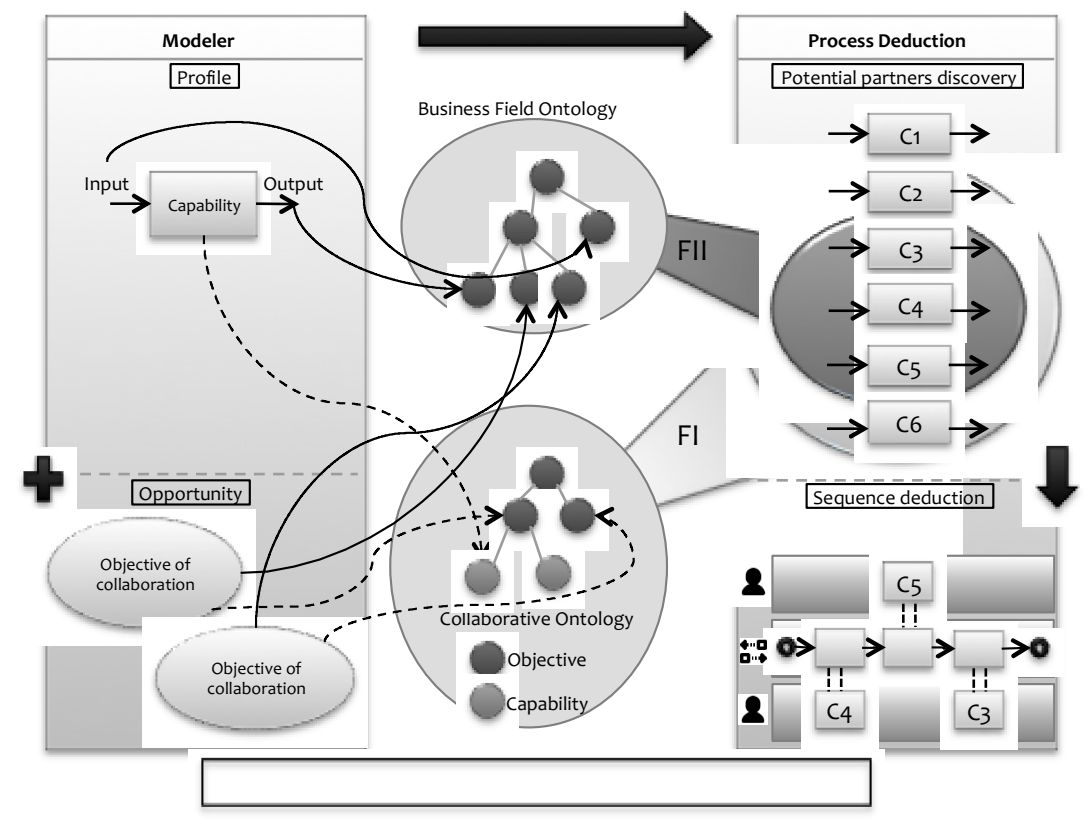

Fig. 1. Functional overview of the OpenPaaS process deduction service

\section{Research Direction towards Functional/Non-functional and Business/Technical Reconciliations}

In [12], [13] and [14], Mu, Boissel-Dallier and then Zribi have written complementary $\mathrm{PhD}$ thesis in the context of the MISE (Mediation Information System Engineering) 
project [15] (cf. Fig. 3). First Mu provides a method to obtain business process cartography from objectives of collaboration and the capabilities of the partners. Then Boissel-Dallier deduces "technical processes" through business/technical reconciliation and obtains potential technical services and their order of execution. Finally, Zribi provides a way to rank and find the best technical services according to nonfunctional criteria.

However in the OpenPaaS context, two observations appear:

1. Non-functional assessment of the collaborative process: OpenPaaS is a "competitive" platform: several partners could offer the same capabilities but with different conveniences (location, cost...). This is a major contribution that should be added to Mu's approach. Moreover, Zribi's works focus essentially on the non-functional assessment of inter-organizational process, however they are situated in the "downstream" phase, which is the assessment of technical services. These works should be adapted to the business process deduction phase.

2. Technical reconciliation: OpenPaaS aims at orchestrating inter-organizational processes. That is why the business processes should be transformed into "technical processes" that can be executed. Boissel-Dallier proposes an approach to achieve this reconciliation: how to switch from 1 to $n$ business services to 1 to $n$ matching technical services. Zribi's PhD thesis comes as a continuation of Boissel-Dallier's works, with non-functionnal reconciliation. However the OpenPaaS context leads to reconsider these separate steps as a unique technical and non-functional reconciliation.

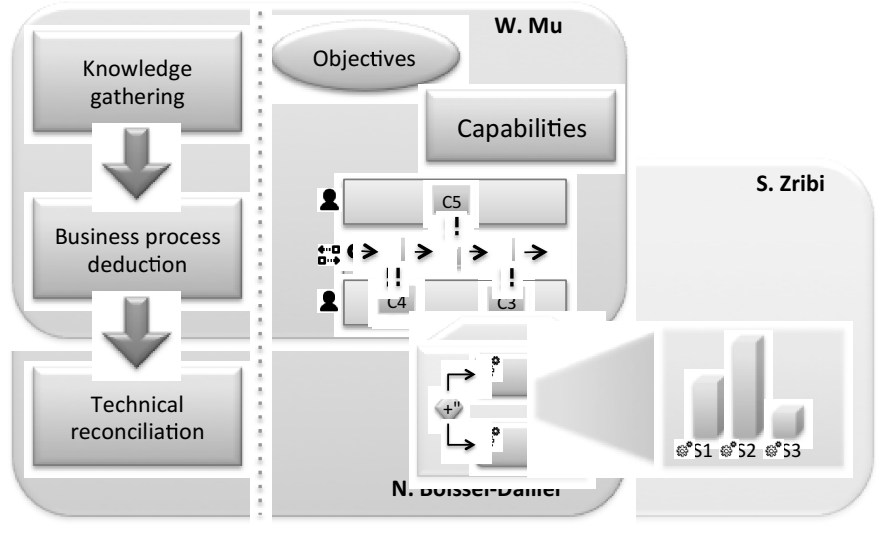

Fig. 2. Contributions in MISE project

\subsection{Functional Versus Non-functional}

As evoked in the previous part, a non-functional assessment of the process should be added to Mu's works. In this regard, a literature review on non-functional factors for the assessment of collaborative networks has already been written [16]. As a result of this study, a list of 33 criteria has emerged, which goal is to assess capabilities the 
most exhaustively possible. However, choosing the best capabilities one per one do not automatically lead the best process. This is why the whole process should be assessed. Since a large amount of potential partners are expected on the OpenPaaS platform, the assessment of all the potential process would be laborious and time-consiuming. An answer to this issue is based on the simultaneous building, assessment of the business processes and consequently selection of the partners.

\subsection{Business Versus Technical}

The business process model would be of no use if it were not for a further orchestration. During the orchestration, the system invokes the technical services corresponding to the business services that have been lately chosen. The difference between business and technical services arises because two different types of users are concerned: (i) some have managerial responsibilities and have often a global view of their company, that is why most of the time they will be led to describe business services; (ii) others have technical responsibilities that give them expert view on technical services. Because of these multiple potential points of view, the assumption is made that at least an organization provides all of the business capabilities that can be shared for inter-organizational collaborations. However zero, one or several technical services can respond to a business capability. It leads to two statements: (i) technical services should be non-functionally annotated as well, (ii) final process could by hybrid, with technical services that can be called during the orchestration and business capabilities if no corresponding technical services have been described.

Along with the functional/non-functional reconciliation, technical services should be selected when building the process.

\subsection{Towards an Ant Colony Optimization (ACO) Algorithm}

In complementary of the method described in Section 3, an algorithm has to be set up to deal with the functional constraints of the process deduction but also the two latter reconciliations. Since a lot of potential solutions are expected, the goal of this algorithm is to find an optimal technical (or hybrid) process.

Moreover, the current approach is based on paths selection: from collaborative objectives to required capabilities in the $\mathrm{CO}$, then from required capabilities to partners' capabilities and finally from partners' capabilities to technical services if they exist.

Although metaheuristic approaches have been widely used in logistics for partner selection for example, they are often based on the assumption that the main steps of the process are already known. In OpenPaaS case, the complexity of the problem comes from the facts that (i) a collaborative objective could be reached by different sets of capabilities and (ii) these capabilities could be provided by different competitive organizations.

It has intuitively led the research towards metaheuristic and more precisely Ant Colony Optimization (ACO). This ACO should indeed allow at efficiently browsing a lot of potential paths, in order to find an optimum. The ACO is based on cycles. On each cycle a certain amount of ants browses the paths and assesses the built solution 
by setting more or less pheromone according to the non-functional annotations of the collaborative objectives. At the end of each cycle, the pheromone evaporates. Finally the optimum path (i.e. the optimum process) is the path that contains the largest quantity of pheromone.

The Fig. 3 brings a global overview of the application of an ACO algorithm in the OpenPaaS context. It introduces the constraints to be respected by the ants when browsing the paths and the behavior they should follow:

- From collaborative objective to required capabilities: the ants browse the CO. They have to discover the capabilities that contributesTo the collaborative objective and should duplicate themselves at each node (since the structure of the ontology states that all the capalibities that contributesTo the objective are complemantary).

- From required capabilities to partners' capabilities: the ants are constrained by the fact that the partners' capabilities should be sameAs/nearBy the require capabilities. At this step, the paths are competing with each other, that is why the ants have to follow random paths.

- From partners' capabilities to technical services: similarly to the previous step, if the technical services exist and have been decently described by the organization, the ants have to browse the technical services that answer to the business capabilities.

Finally the path that still contains most of the pheromone at the end of the cycles represents the optimal process that can be proposed to the broker organization.

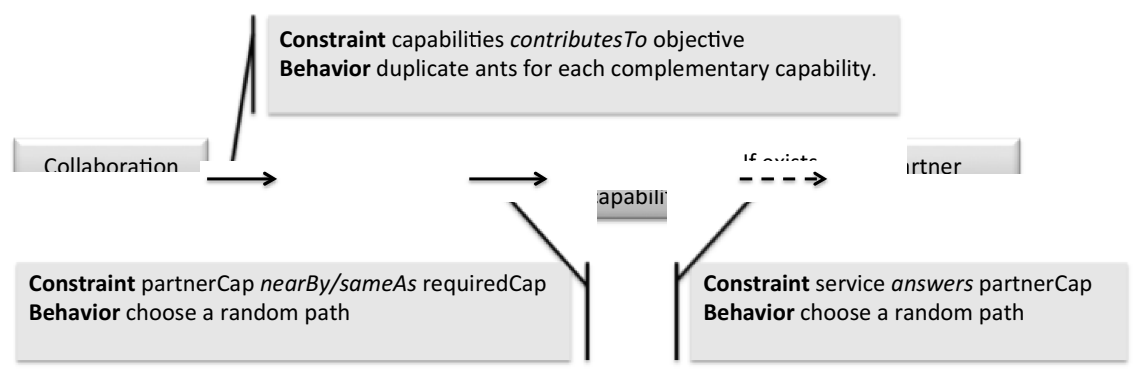

Fig. 3. Overview of the ant colony behavior

\section{$5 \quad$ Conclusion and Perspectives}

This paper presents a method to automatically design inter-organizational business process. From the objectives of the collaboration, we propose a method that (i) establish the list of every required capability to fulfill the objectives, (ii) then discover the corresponding partners and finally (iii) link the partners capabilities with each other to obtain the business process. The main contributions of this article lies on two levels: (i) a semantic-based method to functionally deduce a collaborative business process and (ii) a discussion and new investigations on the simultaneous establishment and 
assessment of a process with business/technical and functional/non-functional reconciliations through an ACO algorithm. Future works have now to be led on these areas of research.

However, the collaborative platform that has been described in this paper suffers from some lacks. First, the question of security and of dissemination of information should be deeply studied: which information organizations would like to share with which others organizations, during design or run time? Moreover this system entirely relies on IT technologies. This means that if the system encounters some technical problems, the supported inter-organizational collaborations could face severe consequences. Some studies oriented towards continuity of service or the data security especially on cloud platforms could be led to enhance the system robustness.

Acknowledgments. The authors wish to thank the French Project OpenPaaS partners for their support.

\section{References}

1. Camarinha-Matos, L.M., Afsarmanesh, H., Galeano, N., Molina, A.: Collaborative networked organizations - Concepts and practice in manufacturing enterprises. Comput. Ind. Eng. 57(1), 46-60 (2009)

2. Byrne, J.A.: The Virtual Corporation. BusinessWeek [Internet] (1993)

3. Bonitasoft - Open Source Workflow \& BPM software, http://fr.bonitasoft. $\mathrm{com} /$

4. Kahua. Kahua Homepage, http: //na1portal .kahua.com/Home

5. Davis, R., Brabander, E.: ARIS Design Platform: Getting Started with BPM, 370 p. Springer (2007)

6. Missbach, M., Stelzel, J., Gardiner, C., Anderson, G., Tempes, M.: SAP Solutions on Public Clouds. In: SAP on the Cloud [Internet], pp. 117-136. Springer, Heidelberg (2013)

7. Accorsi, R.: Business process as a service: Chances for remote auditing. In: IEEE 35th Annual Computer Software and Applications Conference Workshops (COMPSACW) [Internet], pp. 398-403. IEEE (2011)

8. Han, Y.-B., Sun, J.-Y., Wang, G.-L., Li, H.-F.: A cloud-based bpm architecture with userend distribution of non-compute-intensive activities and sensitive data. J. Comput. Sci. Technol. 25(6), 1157-1167 (2010)

9. IFIP-IFAC Task Force on Architectures for Enterprise Integration. GERAM: Generalised Enterprise Reference Architecture and Methodology. Version 1.6.3 (1999)

10. Mu, W., Bénaben, F., Pingaud, H., Boissel-Dallier, N., Lorré, J.-P.: A Model-Driven BPM Approach for SOA Mediation Information System Design in a Collaborative Context. In: 2011 IEEE International Conference on Services Computing (SCC), pp. 747-748 (2011)

11. Kulvatunyou, B., Cho, H., Son, Y.J.: A semantic web service framework to support intelligent distributed manufacturing. Int. J. Knowl.-Based Intell. Eng. Syst. 9(2), 107-127 (2005)

12. Sha, D.Y., Che, Z.H.: Supply Chain Network Design: Partner Selection and Production/Distribution Planning Using a Systematic Model. J. Oper. Res. Soc. 57(1), 52-62 (2006)

13. Malone, T.W., Crowston, K., Herman, G.A.: Organizing business knowledge: the MIT process handbook [Internet]. MIT Press (2003) 
14. United Nations, Statistical Division. International Standard industrial classification of all economic activities (ISIC). United Nations, New York (2008)

15. Mu, W.: Caractérisation métier et logique d'une situation collaborative. INP Toulouse Ecole des Mines d'Albi-Carmaux (2012)

16. Boissel-Dallier, N.: Aide à la conception d'un système d'information de médiation collaboratif: de la cartographie de processus métier au système exécutable. INP Toulouse Ecole des Mines d'Albi-Carmaux (2012)

17. Zribi, S.: La gouvernance SOA pour une approche de conception de Système d'Information de Médiation: réconciliation non-fonctionnelle de services pour mettre en œuvre les processus métier. INP Toulouse - Ecole des Mines d'Albi-Carmaux (2014)

18. Benaben, F., Lauras, M., Truptil, S., Lamothe, J.: MISE 3.0: An Agile Support for Collaborative Situation. In: Camarinha-Matos, L.M., Xu, L., Afsarmanesh, H. (eds.) PRO-VE 2012. IFIP AICT, vol. 380, pp. 645-654. Springer, Heidelberg (2012)

19. Montarnal, A., Lauras, M., Bénaben, F., Lamothe, J.: A Non-functional Framework for Assessing Organizations in Collaborative Networks. In: Mertins, K., Bénaben, F., Poler, R., Bourrières, J.-P. (eds.) Enterprise Interoperability VI [Internet], pp. 251-260. Springer International Publishing (2014) 